\title{
Compartición de noticias en redes sociales. Difusión selectiva y poder social
}

Sharing news in social media. Narrowcasting and social power

Compartilhamento de notícias em redes sociais. Narrowcasting e poder social

Francisco Segado-Boj, Universidad Complutense de Madrid, Madrid, España (fsegado@ucm.es) y Universidad Internacional de la Rioja, La Rioja, España*

María-Ángeles Chaparro-Domínguez, Universidad Complutense de Madrid, Madrid, España (ma.chaparro@ucm.es)

RESUMEN |Este artículo analiza el modo en el que los diferentes objetivos definidos por la teoría del poder social se relacionan con la forma en la que los ciudadanos comparten noticias. Esta teoría postula que las interacciones entre los individuos persiguen alcanzar determinados objetivos por parte de sus interlocutores (recompensa, coerción, legitimación, identificación y demostración de conocimiento). Se busca identificar cómo influyen en las noticias (duras o blandas, emocionales o útiles) que se comparten y dónde (plataformas abiertas asimétricas, como Facebook o Twitter, basadas en modelos de difusión, o entornos cerrados de difusión selectiva como WhatsApp). Se emplea un método de muestreo de experiencias que recogió información acerca de 830 noticias compartidas por 279 participantes adultos en España. Los objetivos más comunes fueron recompensa, legitimación y demostración de conocimiento. Las redes abiertas asimétricas se emplean para compartir noticias con objetivos proselitistas (convencer, persuadir o corregir). Estos objetivos se buscan mediante la difusión de noticias duras, mientras que las noticias blandas se emplean principalmente para recompensar a los contactos, entendido como una manera de estrechar lazos sociales ya existentes. El aspecto emocional (afecto positivo) de las noticias solo adquiere relevancia si el objetivo es la identificación. En el resto de las ocasiones, se comparten preferentemente noticias consideradas útiles.

PALABRAS CLAVE: redes sociales; compartición de noticias; poder social; noticias duras; noticias blandas; afecto positive; utilidad. 
ABSTRACT/This research analyses the way in which news sharing activity diverges according to the different targets defined by social power theory. This theory posits that interactions among individuals are driven by goal-oriented behaviors towards their peers, namely: reward, coercion, legitimation, identification, referential or expertise showcase. Our paper aims to identify how such objectives influence on what news are shared (hard or soft, emotional, or useful) and where are they shared (open asymmetrical, broadcast model, such Facebook or Twitter, or closed, symmetrical, narrowcast platforms like WhatsApp). We applied an experience sample method that gathered information regarding 830 pieces of news shared by 279 adult participants in Spain. Among the main results introduced by our study we point that the most common targets when sharing news were reward, legitimation, and expertise showcase. Open asymmetrical social media were used to sharenews items with proselytizing goals (to convince, persuade or correct). Such goals were pursued by sharing hard news, meanwhile soft news where shared mostly to reward peers, understood as a way of bonding pre-existing social links. The emotional aspect (positive affect) is only relevant when the goal isidentification. In the rest of occasions, usefulnessis the most powerful drier tonews sharing.

KEYWORDS: social media; news sharing; social power; hard news; soft news; positive affect; usefulness.

RESUMO | Este artigo analisa como os diferentes objetivos definidos pela teoria do poder social se relacionam com a forma pela qual os cidadãos comunicam as notícias. Essa teoria do poder social postula que as interações entre os indivíduos buscam atingir determinados objetivos por parte de seus interlocutores (recompensa, coerção, legitimação, identificação e demonstração de conhecimento). Busca-se identificar como esses objetivos influenciam nas notícias (duras ou leves, emocionais ou úteis) que se compartilham e onde (plataformas abertas assimétricas, como Facebook ou Twitter, baseadas em modelos de divulgação ou ambientes fechados de divulgação seletiva como o WhatsApp). É usado um método de amostragem de experiência que coletou informações sobre 830 notícias compartilhadas por 279 participantes adultos na Espanha. Os objetivos mais comuns ao compartilhar uma história eram recompensa, legitimação e demonstração de conhecimento. As redes abertas assimétricas (como Facebook ou Twitter) são usadas para compartilhar notícias para fins de proselitismo (para convencer, persuadir ou corrigir). Esses objetivos são buscados por meio da divulgação de notícias duras, enquanto as notícias leves são utilizadas principalmente para premiar aos contatos, entendido como uma forma de fortalecer os laços sociais existentes. $\mathrm{O}$ aspeto emocional (afeto positivo) das notícias só se torna relevante se o objetivo for a identificação. Em outras ocasiões, notícias consideradas úteis são preferencialmente compartilhadas.

PALAVRAS-CHAVE: mídias sociais; compartilhamento de notícias; poder social; notícias suaves; notícias suaves; afeto positivo; utilidade. 


\section{INTRODUCCIÓN}

Las redes sociales han revolucionado el proceso de consumo y distribución de noticias (Kalsnes \& Larsson, 2019), que abandona un modelo de emisión unidireccional y adopta un paradigma de distribución multidireccional por parte de los usuarios (Noguera-Vivo, 2018). Resulta así vital comprender cómo y por qué los individuos comparten noticias en estos entornos.

Compartir noticias en redes sociales posee un relevante marcado cariz social. Desde el punto de vista de la teoría de los usos y gratificaciones (Katz, 1959), los individuos comparten noticias en estos entornos para lograr dos objetivos: proyectar su imagen a sus contactos e interactuar con ellos (Lee \& Ma, 2012). Es decir, los usuarios comparten noticias fundamentalmente para gestionar su relación con sus contactos. Dado el carácter social de esta actividad, la investigación sobre noticias y redes sociales ha ido incluyendo teorías relacionadas con las relaciones humanas (Duffy \& Ling, 2019). Este estudio sigue esta tendencia, aplicando la teoría del poder social (French Jr. \& Raven, 1959), que conceptualiza las relaciones entre dos individuos según los objetivos que un sujeto (el emisor) intenta obtener de otro (el receptor). Estos objetivos son:

1. Recompensa: la percepción de que el emisor puede conseguir alguna prebenda para el receptor.

2. Coerción: la capacidad del emisor para aplicar algún castigo al receptor.

3. Legitimación: la capacidad de prescribir o justificar comportamientos y actitudes.

4. Identificación: la capacidad de aumentar la identificación entre receptor y emisor.

5. Demostración de conocimiento: la proyección de que el emisor tiene algún conocimiento o habilidad específica.

El objetivo general de este artículo es estudiar la relación entre poder social y compartición de noticias. Se busca identificar cómo los diferentes objetivos de poder social influyen en los tipos de noticias que se comparten y en qué tipo de plataformas.

Estudios previos desde la teoría de usos y gratificaciones (Lee \& Ma, 2012; Hossain et al., 2018; Thompson et al., 2019; Winter et al., 2016) apuntan a que la expectativa de diferentes usos y gratificaciones conduce a distintas actitudes de compartición de noticias. Por ello, de manera simétrica, esperamos que los distintos tipos de poder social motiven diferentes conductas de compartición de noticias. 
Por otro lado, cada red social posee características específicas que influyen en el comportamiento de sus usuarios y, por lo tanto, provocan diferentes efectos (Hasell \& Weeks, 2016) y actitudes (Segado-Boj et al., 2019).

Por ejemplo, Facebook e Instagram fomentan la autopresentación, mientras que Twitter funciona como una plataforma para la búsqueda de información (Boczkowski et al., 2018). Así, los usuarios de distintas plataformas comparten preferentemente distintos tipos de información en uno u otro entorno (Larsson, 2019). Dado que los usuarios no perciben el mismo sentido de influencia cuando se comunican en espacios sociales abiertos que cuando lo hacen en entornos más privados (Oeldorf-Hirsch \& Sundar, 2015) esperamos encontrar diferencias entre el poder social perseguido y el tipo de plataforma en la que se comparten las noticias, específicamente en función de si se tratan de redes sociales abiertas y asimétricas (RAA) y redes sociales cerradas y simétricas (RCS) (Kim \& Ihm, 2019).

En las RAA, los mensajes publicados por el usuario pueden ser vistos por toda la comunidad de sus contactos (seguidores en Twitter o Instagram, amigos en Facebook (Kim \& Ihm, 2019)). En este modelo de emisión un mismo mensaje se difunde a toda una comunidad, sin diferenciar entre individuos específicos (Yang, 2016), lo que conduce frecuentemente al fenómeno del colapso de contexto, es decir, a la confusión de audiencias con diferentes intereses, actitudes o creencias en un mismo grupo general (Gil-López et al., 2018). Esta fusión de seguidores en una misma audiencia amplia y general lleva a los usuarios a aplicar estrategias de gestión de la audiencia (Marwick \& boyd, 2011), entre las que se encuentra la autocensura o la marginación de noticias controvertidas (Kim \& Ihm, 2019).

Las RCS representan un paradigma opuesto a las RAA. Las RCS son creadas por un usuario y a ellas solo pueden unirse usuarios por invitación expresa. Se constituyen como comunidades privadas, no abiertas al público general, por lo que también han sido etiquetadas como redes sociales oscuras (Swart et al., 2018). Sus ejemplos más relevantes son las aplicaciones de mensajería móvil y algunos elementos de las RAA, como los grupos privados de Facebook. Las RCS, siguen, en consecuencia, un modelo de narrowcasting o difusión selectiva (Goncalves et al., 2013), en el que los usuarios distribuyen sus mensajes entre un conjunto reducido y específicamente seleccionado de seguidores (Karapanos et al., 2016).

Estas características pueden ser subjetivas, como la relevancia percibida (Kümpel, 2019), u objetivas, como el tema. Así, ciertos asuntos como la política han demostrado ser especialmente populares en redes sociales (Orellana-Rodriguez \& Keane, 2018).

En cuanto a la percepción de los usuarios, se analizan dos dimensiones subjetivas: utilidad y afecto positivo (Houston et al., 2018). No obstante, el aspecto informativo 
no explica totalmente qué noticias se comparten en redes sociales. Las respuestas emocionales, tales como el disfrute de un artículo, también influyen en este sentido (An et al., 2014). Este estudio mide la respuesta emocional en términos de afecto positivo, es decir, hasta qué punto los usuarios encuentran el mensaje agradable, entretenido y positivo (Houston et al., 2018).

\section{METOdOLOGÍA}

Para alcanzar el objetivo propuesto -determinar cómo influyen los distintos objetivos de poder social en cómo, qué y dónde comparten noticias los usuarioseste estudio toma como unidad de análisis las situaciones en lugar de los actores (Witschge et al., 2018) y emplea un método de muestreo de experiencias (MME), en el que los participantes contestan en determinados momentos aleatorios distintas preguntas acerca de alguna experiencia (Kubey et al., 1996).

La primera pregunta de investigación (PI1) es: ¿Cuáles son los objetivos de poder social más esperados cuando se comparten noticias en redes sociales? La segunda pregunta de investigación (PI2) consulta sobre ¿Qué tipo de plataformas escogen los usuarios en función del poder social que quieren ejercer cuando comparten una noticia? Como ya se ha mencionado, las expectativas e intenciones de los usuarios influyen en las características de contenido que comparten en redes sociales (Johnson \& Ranzini, 2018). Por ello, se plantea la pregunta (PI3): ¿Qué contenido eligen compartir los usuarios en función del poder Social que esperan ejercer al compartir una noticia? Dado que la utilidad es uno de los valores que predicen la viralidad del contenido en redes sociales (Knobloch-Westerwick \& Kleinman, 2012), se plantea la pregunta de investigación (PI4): ¿Qué relación existe entre la percepción de utilidad de una noticia y el tipo de poder social que se quiere ejercer al compartir esa noticia en redes sociales?

Finalmente, la pregunta de investigación (PI5)aborda ¿Qué relación existe entre el afecto positivo respecto de una noticia y el poder social que se quiere ejercer cuando se comparte una noticia en redes sociales?

Para responder a las interrogantes anteriores, se contó con 300 voluntarios entre 18 y 39 años, reclutados por la empresa demoscópica Societae. Los participantes tuvieron que ofrecer inicialmente una serie de información sociodemográfica (ver tabla 1). Veintiún participantes no completaron el cuestionario inicial y no fueron incluidos en la muestra final $(\mathrm{n}=279)$. Las respuestas se recogieron del 16 de noviembre al 12 de diciembre de 2018. Se recogió un total de 830 experiencias válidas, es decir de noticias compartidas por los usuarios y su evaluación (ver tablas 2, 4 y 5). 


\begin{tabular}{|c|c|c|c|}
\hline & & $\mathbf{n}$ & $\%$ \\
\hline \multirow{3}{*}{ Género } & Masculino & 109 & 39,07 \\
\hline & Femenino & 167 & 59,86 \\
\hline & Prefiero no decirlo & 3 & 1,08 \\
\hline \multirow{5}{*}{ Educación } & Básica & 5 & 1,79 \\
\hline & Secundaria & 40 & 14,34 \\
\hline & Profesional & 71 & 25,45 \\
\hline & Universitaria & 162 & 58,07 \\
\hline & Doctorado & 1 & 0,36 \\
\hline \multirow{12}{*}{ Ingresos mensuales } & Sin ingresos & 9 & 3,23 \\
\hline & $€ 0<300$ & 6 & 2,15 \\
\hline & $€ 301<600$ & 14 & 5,02 \\
\hline & $€ 601<900$ & 17 & 6,09 \\
\hline & $€ 0<1.200$ & 36 & 12,90 \\
\hline & $€ 1.201<1.800$ & 67 & 24,01 \\
\hline & $€ 1.801<2.400$ & 46 & 16,49 \\
\hline & $€ 2.401<3.000$ & 38 & 13,62 \\
\hline & $€ 3.001<4.500$ & 29 & 10,39 \\
\hline & $€ 4.501<6.000$ & 7 & 2,51 \\
\hline & $€>6.000$ & 2 & 0,72 \\
\hline & Prefiero no decirlo & 8 & 2,87 \\
\hline
\end{tabular}

Tabla 1. Características sociodemográficas de la muestra

Fuente: Elaboración propia.

En tres momentos ( 16 de noviembre, 24 de noviembre y 2 de diciembre), los usuarios recibieron por correo electrónico un cuestionario con preguntas acerca de la última noticia que hubieran compartido en redes sociales, mensajería instantánea o cualquier otro canal.

La tabla 2 indica las opciones presentadas a los usuarios acerca de la plataforma en la que compartieron la noticia. Las opciones 1-2 se consideraron RAA y las 3-6, RCS. Las opciones 7-8 no consideraron dado el escaso número de respuestas. 
n

$\%$

1. En alguna red social (Twitter, Facebook, Instagram...) como una publicación para que la puedan ver todos mis contactos en general.

RAA

2. En redes sociales, pero me he dirigido a una persona específica, etiquetándole en Facebook o Instagram o mencionándole en Twitter. Aun así, el mensaje era público y lo podían ver todos mis contactos.

3. En un grupo privado o comunidad restringida de Facebook o cualquier otra red social, de modo que solo lo puedan ver los miembros de ese grupo o comunidad.

RCS

\section{En un grupo privado de WhatsApp, Line, Telegram o Facebook Messenger.}

5. Se lo he enviado a un contacto concreto mediante mensajería instantánea (WhatsApp, Line, Telegram o Facebook Messenger).

o oacebook Mess
252 30,36
6. Por correo electrónico, con una persona o grupo de personas determinado.

$6 \quad 0,72$

7. Por correo electrónico, de manera masiva a un gran grupo de personas (más de 50).

0

8. Otro.

9

1,09

Tabla 2. Plataformas en las que se compartieron las noticias

Fuente: Elaboración propia.

Los participantes tuvieron que indicar el enlace de la noticia que compartieron. El cuestionario mencionaba un concepto amplio de noticia, que no requería que fuese publicado por un medio convencional. En el caso de que la noticia no incluyera un enlace, los usuarios tuvieron que indicar el titular de la noticia. A partir de estos enlaces y noticias, se categorizaron las historias de acuerdo a su tema (Kilgo et al., 2018) (tabla 3).

La codificación corrió a cargo de uno de los autores, por lo que no se calculó ningún índice de fiabilidad interjueces. Dada la elevada dispersión de temas, las categorías se agruparon en cuatro grandes bloques temáticos, como se indica en la columna de la izquierda de la tabla 3. 


\begin{tabular}{|c|c|c|c|}
\hline & Tema & $n$ & $\%$ \\
\hline \multirow{8}{*}{ Noticias duras } & Relaciones internacionales & 45 & 5,74 \\
\hline & Ejército / Defensa & 2 & 0,26 \\
\hline & Gobierno & 102 & 13,01 \\
\hline & Crimen / Sucesos & 128 & 16,33 \\
\hline & Economía / Negocios / Finanzas & 38 & 4,85 \\
\hline & Derechos civiles & 28 & 3,57 \\
\hline & Medioambiente & 63 & 8,04 \\
\hline & Religión & 3 & 0,38 \\
\hline \multirow{3}{*}{ Noticias blandas } & Deportes & 46 & 5,87 \\
\hline & Entretenimiento & 96 & 12,25 \\
\hline & Estilo de vida / Salud & 67 & 8,55 \\
\hline \multirow{2}{*}{ Cultura } & Educación & 69 & 8,80 \\
\hline & Ciencia/Tecnología & 25 & 3,19 \\
\hline \multirow[t]{2}{*}{ Otros } & Otros & 72 & 9,18 \\
\hline & Total & 784 & 100 \\
\hline
\end{tabular}

*Nota: 46 ítems quedaron fuera del análisis, dado que no ofrecieron suficiente información como para asignarles una categoría.

Tabla 3. Temas considerados en el análisis de contenido

Fuente: Elaboración propia.

\section{Medidas}

Se elaboró una serie de preguntas para identificar los aspectos del poder social desarrollados en Lukes (2005) a partir de los cinco objetivos formulados por French Jr. y Raven (1959). Las preguntas se plantearon en formato Likert y los usuarios debían expresar su acuerdo con las afirmaciones planteadas ( $1=$ nada de acuerdo, $5=$ totalmente de acuerdo), como se ve en la tabla 4 .

Recompensa se divide en tres percepciones diferentes, interpretando que la prebenda puede consistir en ayudar a promover una actividad o evento, ofrecer información útil o divertir al receptor. Legitimación incluye expresión de opinión, apoyar un argumento y prescribir actitudes o ideas. Identificación se divide en dos aspectos: relacional y conversacional. Demostración de conocimiento se divide en proyección de las habilidades y educacional. Por último, la coerción se interpreta en este estudio desde el punto de vista del receptor, entendida como la evitación de conflicto o castigos por parte de otro (tabla 4). 
Etiqueta

Recompensa

(evento)

Recompensa

(info. útil)
Afirmación. "He compartido esta

noticia...
Media DE

$3,31 \quad 1.52$ ...para ayudar a dar a conocer un evento o una actividad. ...porque creo que a una persona o a

un grupo de gente les iba a venir bien disponer de esa información.

...porque he pensado que esta noticia iba a divertir o le iba a parecer curiosa a una persona o a un grupo de personas.

Legitimación

(mi opinión) ...para expresar una idea, un punto de vista o mis sentimientos sobre un tema o acontecimiento.

...para reforzar mis argumentos sobre una cuestión.

Legitimación
(influencia)

...porque me ayuda a sentirme más

Identificación

cerca de una persona o de un grupo de

2,31

(relacional) personas.

Identificación

(conversacional) ...porque me ayuda a abrir una conversación.

Demostración de conocimiento (mis habilidades)

...porque quería demostrar mis conocimientos sobre un asunto o mi dominio sobre un tema.

Demostración de conocimiento (educativo) ...para ayudar a la gente a entender mejor un asunto o para educarles.

Coerción

\section{Tabla 4. Medidas de la expectativa de poder social}

Fuente: Elaboración propia.

El afecto positivo $(\alpha=0,86)$ y la utilidad $(\alpha=0,85)$ se midieron con las preguntas tipo Likert ( $1=$ nada de acuerdo, 5 = totalmente de acuerdo) indicadas en la tabla 5 (Houston et al., 2018). 


\begin{tabular}{cc}
\hline Afecto positivo & Me ha gustado el contenido. \\
\cline { 2 - 2 } & He disfrutado con el contenido. \\
\hline El contenido es positivo. \\
Et contenido es entretenido. \\
\hline $\begin{array}{c}\text { El contenido es valioso (en general, para mí, } \\
\text { para mis amigos o para la sociedad). }\end{array}$ \\
El contenido es bueno y de calidad. \\
El contenido es útil. \\
\hline El contenido me ha ayudado a estar informado. \\
\hline El contenido es relevante para mi vida.
\end{tabular}

Tabla 5. Medidas para la percepción de las noticias compartidas

Fuente: Elaboración propia.

\section{Variables de control}

Se consideraron aspectos socioeconómicos que afectan al uso general de redes sociales, como la edad, el estatus económico (Lottridge \& Bentley, 2018) y la educación (Holton et al., 2015).

Siguiendo las recomendaciones de Segado-Boj y sus colegas (2019), el modelo incluye los rasgos de personalidad de los usuarios. Específicamente, se midió la extroversión $(\alpha=0,8)$ y la apertura a experiencias $(\alpha=0,67)$, puesto que son los rasgos con mayor influencia en la compartición de noticias en redes sociales (Liu \& Campbell, 2017). La extroversión se refiere a la capacidad de una persona de ser sociable y la apertura a experiencias implica la disposición de un individuo a considerar perspectivas alternativas y ser curioso intelectualmente (Ross et al., 2009). Ambos constructos se midieron utilizando instrumentos previos (Gil De Zúñiga et al., 2017), basados en preguntas tipo Likert (1= nada de acuerdo, 5 = totalmente de acuerdo) (tabla 6).

La orientación política también provoca diferencias en el tipo de información que los usuarios comparten en redes sociales (Guess et al., 2019). Similarmente, el extremismo político se ha vinculado a una frecuencia más intensa de compartición de noticias (Kalogeropoulos et al., 2017). Los participantes tuvieron que identificarse en una escala ideológica de 8 puntos $(0=$ extrema izquierda, $7=$ extrema derecha; promedio $=3,80, \mathrm{DE}=1,14)$. Esta respuesta se recodificó posteriormente para medir el extremismo. A los rangos extremos de la escala ( 0 y 7) se les atribuyó un máximo de 4 , y a aquellos en la parte central (3 y 4 ) un mínimo de 1 (promedio= 1,$48 ; \mathrm{DE}=0,74$ ).

El orden de las preguntas sobre poder social, afecto positivo, utilidad percibida, extraversión y apertura a la experiencia se presentó aleatorizado en cada cuestionario. 


\begin{tabular}{|c|c|}
\hline \multirow{6}{*}{ Apertura a experiencias } & "Me resulta difícil imaginar cosas" (recodificada). \\
\hline & "No estoy interesado en nuevas ideas" (recodificada). \\
\hline & "No me gusta probar cosas nuevas" (recodificada). \\
\hline & "Suelo tener muchas ideas". \\
\hline & $\begin{array}{l}\text { "Me lleva mucho tiempo aprender cualquier cosa nueva" } \\
\text { (recodificada). }\end{array}$ \\
\hline & "Entiendo las cosas rápidamente". \\
\hline \multirow{6}{*}{ Extroversión } & "Me gusta arrancar conversaciones". \\
\hline & "No me gusta hablar a un grupo de personas" (recodificada). \\
\hline & "Me siento cómodo presentándome a gente que no conozco". \\
\hline & "Me siento tímido cuando estoy con desconocidos" (recodificada). \\
\hline & "Hablo con diferentes personas en eventos y acontecimientos". \\
\hline & "Me resulta difícil acercarme a otras personas" (recodificada). \\
\hline
\end{tabular}

Tabla 6. Medidas empleadas para rasgos de personalidad

Fuente: Elaboración propia.

\section{Análisis de los datos}

Para el análisis se utilizó el lenguaje informático R. Se aplicaron pruebas ANOVA para detectar diferencias entre el tipo de red social (RAA o RCS) en la que se comparte la noticia y los distintos objetivos de poder social (PI2). También se emplearon pruebas ANOVA para identificar diferencias a raíz de los temas de las noticias (PI3). Los resultados se muestran en las tablas 7 y 8. Dada la elevada disparidad de conjuntos contemplados, se aplicó la prueba post hoc Bonferroni (ver tablas 8 y 10). Por último, se aplicó un análisis de regresión múltiple para comprobar si la percepción del mensaje (PI4) influía en la intención de cada objetivo de poder social considerado. En todos los casos (tablas 7-12) se destacan en negrita los valores en los que existen diferencias, al igual que las asociaciones significativas $(\mathrm{p}<0,05)$. Los resultados ANOVA desagregados para cada tema específico recogido en la tabla 3 se ofrecen como material adicional.

\section{RESULTADOS}

\section{Diferencias entre el tipo de red social y el objetivo de poder social}

Se encontraron diferencias significativas (tabla 7) entre el tipo de objetivos de poder social perseguido y la plataforma en la que se comparte la noticia. 


\begin{tabular}{ccc} 
Objetivos de poder social & F & P \\
\hline Recompensa (evento) & 0,571 &, 565 \\
\hline Recompensa (info. útil) & 1,357 &, 258 \\
\hline Recompensa (diversion) & 1,824 &, 162 \\
\hline Coerción & 0,654 &, 520 \\
\hline Legitimación (mi opinión) & 16,246 & $<, 001$ \\
\hline Legitimación (refuerzo argumental) & 3,485 &, 031 \\
\hline Legitimación (influencia) & 11,787 & $<, 001$ \\
\hline Identificación (relacional) & 0,769 &, 464 \\
\hline Identificación (conversacional) & 2,627 &, 073 \\
\hline Demostración de conocimiento (mis habilidades) & 1,305 &, 272 \\
\hline Demostración de conocimiento (educativo) & 12,265 & $<, 001$ \\
\hline
\end{tabular}

Tabla 7. Diferencias entre el tipo de objetivos de Poder Social perseguido y el tipo de plataforma en el que se comparte la noticia

Fuente: Elaboración propia.

$\begin{array}{llll}\text { Diff } & \text { Lwr } & \text { Upr } & \text { P adj }\end{array}$

Legitimación (mi opinión)

\begin{tabular}{c|c|c|c|c}
\hline RAA - RCS & 0,544 & 0,263 & 0,826 & $<, 001$ \\
\hline RCS - Otros & 0,352 & 1,707 & 1,004 & 1 \\
\hline RAA - Otros & 0,193 & $-1,161$ & 1,547 & 1
\end{tabular}

Legitimación (refuerzo argumental)

\begin{tabular}{c|c|c|c|c}
\hline RAA - RCS & 0,249 & $-0,047$ & 0,545 &, 041 \\
\hline RCS - Otros & 0,562 & $-0,863$ & 1,987 &, 738 \\
\hline RAA - Otros & 0,313 & $-1,114$ & 1,740 & 1 \\
\hline
\end{tabular}

Legitimación (influencia)

\begin{tabular}{c|c|c|c|c}
\hline RAA - RCS & 0,477 & 0,180 & 0,773 & $<, 001$ \\
\hline RCS - Otros & $-0,788$ & $-2,219$ & 0,642 &, 315 \\
\hline RAA - Otros & $-0,312$ & $-1,740$ & 1,117 & 1
\end{tabular}

Demostración de conocimiento (educativo)

\begin{tabular}{c|c|c|c|c}
\hline RAA - RCS & 0,476 & 0,190 & 0,762 & $<, 001$ \\
\hline RCS - Otros & $-0,574$ & $-1,951$ & 0,803 & 0,661 \\
\hline RAA - Otros & $-0,098$ & $-1,473$ & 1,278 & 1 \\
\hline
\end{tabular}

Tabla 8. Análisis post hoc (Bonferroni) de diferencias entre los objetivos de poder social perseguidos y el tipo de red social

Fuente: Elaboración propia 
El análisis post hoc (tabla 8) identifica diferencias a favor de las RAA, es decir, del modelo de emisión masiva de redes como Facebook, Twitter u otras en comparación con los paradigmas de emisión selectiva como WhatsApp o Facebook Messenger.

También se encontraron diferencias significativas (tabla 9) entre el tipo de objetivo del poder social y el tema de la noticia compartida.

\begin{tabular}{ccc} 
Objetivos de poder social & $\mathbf{F}$ & $\mathbf{P}$ \\
\hline Recompensa (evento) & 8,48 & $<, 001$ \\
\hline Recompensa (info útil) & 2,774 &, 040 \\
\hline Recompensa (diversión) & 17,97 & $<, 001$ \\
\hline Coercitivo & 3,197 &, 023 \\
\hline Legitimación (mi opinión) & 3,003 &, 030 \\
\hline Legitimación (refuerzo argumental) & 2,133 &, 095 \\
\hline Legitimación (influencia) & 7,226 & $<, 001$ \\
\hline Identificación (relacional) & 4,673 &, 003 \\
\hline Identificación (conversacional) & 0,924 &, 429 \\
\hline Demostración de conocimiento (mis habilidades) & 0,127 &, 944 \\
\hline Demostración de conocimiento (educativo) & 11,67 & $<, 001$ \\
\hline
\end{tabular}

Tabla 9. Diferencias entre los objetivos del poder social perseguido y el tema de las noticias compartidas

Fuente: Elaboración propia.

El análisis post hoc de los casos significativos (tabla 10) arroja diferencias a favor de las noticias duras y de las noticias blandas. La preferencia hacia otros temas fue menos común.

En los casos sobre Recompensa (evento) no se encuentran diferencias significativas entre noticias blandas y duras. La preferencia es hacia Cultura y Otros temas, en detrimento de noticias blandas y duras. Recompensa (diversión) muestra un patrón similar, con una menor tendencia a noticias duras, menos prominentes que el resto de los asuntos. En Legitimación (mi opinión), Legitimación (influencia) y Demostración de conocimiento (educativo), las noticias blandas son menos comunes que las duras.

En Identificación (relacional), solo se encuentran diferencias entre la categoría Otros, que predomina sobre noticias duras. Un hecho similar ocurre en Coerción. Esto podría interpretarse como que los objetivos de Identificación y Coerción son independientes de la agenda mediática de la actualidad. 


\section{Recompensa (evento)}

\begin{tabular}{ccccc} 
& Diff & Lwr & Upr & P adj \\
\hline Noticias blandas - Noticias duras & $-0,013$ & $-0,416$ & 0,388 & 1 \\
\hline Cultura - Noticias duras & 0,585 & 0,044 & 1,126 &, $004^{* *}$ \\
\hline Otros - Noticias duras & 0,736 & 0,132 & 1,340 & $<, 001^{* * *}$ \\
\hline Cultura - Noticias blandas & 0,598 & 0,012 & 1,185 &, $008^{* *}$ \\
\hline Otros - Noticias blandas & 0,749 & 0,103 & 1,395 &, $002^{* *}$ \\
\hline Otros - Cultura & 0,151 & $-0,589$ & 0,891 & 1
\end{tabular}

\section{Recompensa (diversión)}

\begin{tabular}{ccccc} 
& Diff & Lwr & Upr & P adj \\
\hline Noticias blandas - Noticias duras & 0,731 & 0,361 & 1,101 & $<, 001^{* * *}$ \\
\hline Cultura - Noticias duras & 0,824 & 0,327 & 1,321 & $<, 001^{* * *}$ \\
\hline Otros - Noticias duras & 0,464 & $-0,092$ & 1,019 &, 052 \\
\hline Cultura - Noticias blandas & 0,093 & $-0,447$ & 0,633 & 1 \\
\hline Otros - Noticias blandas & $-0,268$ & $-0,862$ & 0,327 &, 934 \\
\hline Otros - Cultura & $-0,360$ & $-1,042$ & 0,320 &, 572
\end{tabular}

\section{Coerción}

\begin{tabular}{ccccc} 
& Diff & Lwr & Upr & P adj \\
\hline Noticias blandas - Noticias duras & 0,042 & $-0,225$ & 0,308 & 1 \\
\hline Cultura - Noticias duras & 0,093 & $-0,265$ & 0,451 & 1 \\
\hline Otros - Noticias duras & 0,389 & $-0,011$ & 0,790 &, $013^{*}$ \\
\hline Cultura - Noticias blandas & 0,051 & $-0,338$ & 0,440 & 1 \\
\hline Otros - Noticias blandas & 0,348 & $-0,080$ & 0,776 &, 063. \\
\hline Otros - Cultura & 0,296 & $-0,194$ & 0,787 &, 342
\end{tabular}

Legitimación (mi opinión)

\begin{tabular}{ccccc} 
& Diff & Lwr & Upr & P adj \\
\hline Noticias blandas - Noticias duras & $-0,339$ & $-0,711$ & 0,034 &, $025^{*}$ \\
\hline Cultura - Noticias duras & $-0,167$ & $-0,669$ & 0,334 & 1 \\
\hline Otros - Noticias duras & $-0,262$ & $-0,822$ & 0,298 &, 842 \\
\hline Cultura - Noticias blandas & 0,172 & $-0,373$ & 0,716 & 1 \\
\hline Otros - Noticias blandas & 0,077 & $-0,522$ & 0,676 & 1 \\
\hline Otros - Cultura & $-0,095$ & $-0,782$ & 0,591 & 1
\end{tabular}




\section{Legitimación (influencia)}

\begin{tabular}{ccccc} 
& Diff & Lwr & Upr & P adj \\
\hline Noticias blandas - Noticias duras & $-0,552$ & $-0,940$ & $-0,164$ & $<, 001^{* * *}$ \\
\hline Cultura - Noticias duras & $-0,272$ & $-0,794$ & 0,250 &, 6061 \\
\hline Otros - Noticias duras & $<0,001$ & $-0,583$ & 0,584 & 1 \\
\hline Cultura - Noticias blandas & 0,281 & $-0,286$ & 0,847 &, 7116 \\
\hline Otros - Noticias blandas & 0,553 & $-0,071$ & 1,176 &, $032^{*}$ \\
\hline Otros - Cultura & 0,272 & $-0,443$ & 0,987 & 1
\end{tabular}

Identificación (relacional)

\begin{tabular}{ccccc} 
& Diff & Lwr & Upr & P adj \\
\hline Noticias blandas - Noticias duras & 0,267 & $-0,082$ & 0,617 &, 096 \\
\hline Cultura - Noticias duras & 0,196 & $-0,274$ & 0,666 & 1 \\
\hline Otros - Noticias duras & 0,550 & 0,0250 & 1,075 &, $006^{* *}$ \\
\hline Cultura - Noticias blandas & $-0,071$ & $-0,581$ & 0,439 & 1 \\
\hline Otros - Noticias blandas & 0,283 & $-0,279$ & 0,844 &, 673 \\
\hline Otros - Cultura & 0,354 & $-0,290$ & 0,998 &, 498
\end{tabular}

\section{Demostración de conocimiento (educativo)}

\begin{tabular}{ccccc} 
& Diff & Lwr & Upr & P adj \\
\hline Noticias blandas - Noticias duras & $-0,684$ & $-1,054$ & $-0,314$ & $<, 001^{* * *}$ \\
\hline Cultura - Noticias duras & $-0,084$ & $-0,581$ & 0,413 & 1 \\
\hline Otros - Noticias duras & $-0,213$ & $-0,769$ & 0,342 & 1 \\
\hline Cultura - noticias blandas & 0,600 & 0,060 & 1,140 &, $003^{* *}$ \\
\hline Otros - Noticias blandas & 0,471 & $-0,123$ & 1,065 &, 075 \\
\hline Otros - Cultura & $-0,129$ & $-0,810$ & 0,552 & 1 \\
\hline
\end{tabular}

*Nota: códigos de significatividad: $0^{\mid * * * 1} 0,001^{1 * * 1} 0,01^{\mid * 1} 0,05^{\prime \prime} .0,1^{\prime \prime} 1$

Tabla 10. Análisis post hoc (Bonferroni) de diferencias entre los objetivos del poder social y el tema de las noticias compartidas

Fuente: Elaboración propia.

\section{Percepción de las noticias y poder social}

La regresión lineal múltiple indica un efecto significativo del modelo propuesto sobre la evaluación de afecto positivo $(F(18,811)=16,17, \mathrm{p}<, 001, \mathrm{R} 2=, 26)$. El análisis posterior de los predictores individuales indica que Recompensa (diversión), Identificación (relacional) e Identificación (conversacional) ejercen una influencia significativa sobre el afecto positivo (tabla 11). 


\begin{tabular}{|c|c|c|c|c|}
\hline & Estimate & Std. Error & $\mathrm{t}$ value & $\operatorname{Pr}(>|t|)$ \\
\hline (Intersección) & 6,221 & 1,510 & 4,120 & $<, 001^{* * *}$ \\
\hline Recompensa (evento) & 0,149 & 0,094 & 1,586 & ,113 \\
\hline Recompensa (info. útil) & 0,172 & 0,135 & 1,276 & ,202 \\
\hline Recompensa (diversión) & 1,358 & 0,100 & 13,614 & $<, 001^{* * *}$ \\
\hline Legitimación (mi opinión) & 0,040 & 0,126 & 0,320 & ,749 \\
\hline Legitimación (refuerzo argumental) & $-0,058$ & 0,128 & $-0,456$ & ,648 \\
\hline Legitimación (influencia) & $-0,158$ & 0,122 & $-1,296$ & 195 \\
\hline Identificación (relacional) & 0,488 & 0,124 & 3,920 & $<, 001^{* * *}$ \\
\hline Identificación (conversacional) & $-0,345$ & 0,125 & $-2,757$ &, $006^{* *}$ \\
\hline $\begin{array}{c}\text { Demostración de conocimiento (mis } \\
\text { habilidades) }\end{array}$ & 0,191 & 0,141 & 1,355 & 176 \\
\hline Demostración de conocimiento (educativo) & 0,081 & 0,132 & 0,614 &, 539 \\
\hline Coerción & 0,017 & 0,151 & 0,112 & 911 \\
\hline Edad & 0,043 & 0,024 & 1,829 & ,068 \\
\hline Ingresos & $-0,072$ & 0,061 & $-1,173$ & ,241 \\
\hline Ideología & 0,129 & 0,128 & 1,008 & ,314 \\
\hline Apertura a la experiencia & 0,014 & 0,046 & 0,302 & ,763 \\
\hline Extroversión & $-0,002$ & 0,031 & $-0,070$ & 944 \\
\hline Educación & $-0,108$ & 0,175 & $-0,616$ &, 538 \\
\hline Extremismo ideológico & 0,325 & 0,194 & 1,674 & 094 \\
\hline
\end{tabular}

*Nota: códigos de significatividad: $0^{(* * *)} 0,001^{(* *)} 0,01^{(*)} 0,05^{\prime \prime}$. 0,1 ' 1

Tabla 11. Resultados de regresión para la evaluación de afecto positivo

Fuente: Elaboración propia.

En cuanto a la utilidad, la prueba de regresión muestra un efecto significativo del modelo $(F(18,811)=30.4, \mathrm{p}<, 001, \mathrm{R} 2=.40)$. Recompensa (evento), Recompensa (info. útil), Legitimación (mi opinión) y Demostración de conocimiento (educativo) funcionan como predictores significativos (tabla 12); es decir, cuanto más relevante es la búsqueda de esos objetivos, mayor es la evaluación del afecto positivo de la noticia compartida. 


\begin{tabular}{|c|c|c|c|c|}
\hline & Estimate & Std. Error & $t$ value & $\operatorname{Pr}(>|t|)$ \\
\hline (Intercept) & 8,710 & 1,346 & 6,472 & $<, 001 * * *$ \\
\hline Recompensa (evento) & 0,477 & 0,084 & 5,716 & $<, 001^{* * *}$ \\
\hline Recompensa (info útil) & 1,373 & 0,120 & 11,425 & $<, 001^{* * *}$ \\
\hline Recompensa (diversión) & $-0,056$ & 0,089 & $-0,628$ &, 531 \\
\hline Legitimación (mi opinión) & 0,321 & 0,112 & 2,859 &, $004^{* *}$ \\
\hline Legitimación (refuerzo argumental) & $-0,024$ & 0,114 & $-0,206$ & 837 \\
\hline Legitimación (influencia) & 0,151 & 0,109 & 1,393 & 164 \\
\hline Identificación (relacional) & $-0,035$ & 0,111 & $-0,316$ & ,752 \\
\hline Identificación (conversacional) & $-0,052$ & 0,111 & $-0,468$ & ,640 \\
\hline $\begin{array}{l}\text { Demostración de conocimiento } \\
\text { (mis habilidades) }\end{array}$ & 0,016 & 0,126 & 0,124 & 901 \\
\hline Demostración de conocimiento (educativo) & 0,727 & 0,118 & 6,189 & $<, 001^{* * *}$ \\
\hline Coerción & $-0,183$ & 0,134 & $-1,361$ & ,173 \\
\hline Edad & $-0,009$ & 0,021 & $-0,413$ & ,680 \\
\hline Ingresos & 0,027 & 0,055 & 0,500 & ,617 \\
\hline Ideología & $-0,058$ & 0,114 & $-0,506$ & 612 \\
\hline Apertura a la experiencia & $-0,005$ & 0,041 & $-0,126$ & 900 \\
\hline Extroversión & 0,030 & 0,028 & 1,081 & ,280 \\
\hline Educación & $-0,059$ & 0,156 & $-0,380$ & ,704 \\
\hline Extremismo ideoloógico & 0,122 & 0,173 & 0,707 & ,480 \\
\hline
\end{tabular}

*Nota: códigos de significatividad: $0^{(* *)} 0,001^{(* *)} 0,01^{(*)} 0,05$ '. 0,1 ' 1

Tabla 12. Resultados de regresión para la evaluación de afecto positivo

Fuente: Elaboración propia.

\section{DISCUSIÓN Y CONCLUSIONES}

Los objetivos de poder social más comunes (PI1) fueron Legitimación y Recompensa (tabla 4). Los usuarios comparten contenidos con fines proselitistas (convencer, persuadir o corregir), pero también para provocar gratitud o reconocimiento en sus contactos. Por tanto, los datos revelan una dualidad entre el proselitismo y la ofrenda en las motivaciones de los usuarios al compartir noticias en sus redes sociales. 
La respuesta a la PI2 (tabla 8) señala que la Legitimación, en todas sus facetas (opinión personal, refuerzo argumental e influencia), se busca principalmente compartiendo noticias en RAA como Facebook o Twitter. Así, esa compartición proselitista recién mencionada explica el dominio del contenido político y valorativo en este tipo de entornos (Lottridge \& Bentley, 2018). Las RAA se convierten en auditorios donde los usuarios lanzan sus proclamas, apoyados en las noticias que comparten.

En RCS, por el contrario, el objetivo persuasivo no resulta predominante, lo que puede explicarse de varias formas. Como estas redes pueden organizarse en torno a experiencias o intereses comunes específicos -por ejemplo, grupos de compañeros de trabajo- (Swart et al., 2018), el proselitismo y podrían, pues, ser interpretados como actitudes impropia en estos entornos. Así, los esfuerzos para divulgar ideas se dirigen a una audiencia más diversa y heterogénea en las RAA. En este sentido, compartir noticias con el objetivo de defender una posición política en RAA sería visto como una práctica menos arriesgada y agresiva, al no ir dirigida a grupos específicos de individuos, sino a un destinatario más indefinido y genérico. El colapso de contexto estimularía el proselitismo y no lo frenaría.

Como explicación alternativa, las RCS están formadas habitualmente por contactos más próximos como amigos o familiares (Waterloo et al., 2018). Consecuentemente, quizás los usuarios esperan que sus contactos en RCS muestren ideologías más próximas que sus contactos en RAA. Este hecho implicaría que los usuarios entienden que sus contactos más cercanos no necesitan ser convencidos, al contrario que aquellos con los que se experimenta menos proximidad.

El empleo de RAA para la difusión de argumentos políticos puede explicar parcialmente el hartazgo de un sector de los usuarios de redes sociales y su migración hacia RCS donde, como señala el estudio, este contenido de carácter proselitista se comparte en menor medida.

Al contrastar el objetivo del poder social perseguido y los temas de las noticias compartidas (PI3), la Recompensa se orienta hacia noticias blandas y la Legitimización y Demostración hacia noticias duras (tabla 10). Este hecho confirma que las noticias duras no se comparten para informar, sino como argumento o herramienta para el combate ideológico. La faceta más amigable de la compartición de noticias (Recompensa) se persigue por el contrario mediante las noticias blandas. La información alejada de cuestiones polémicas como la política resulta más efectiva para cumplir ese objetivo gratificante al que se destinan, de acuerdo con nuestros resultados. 
En cuanto a la evaluación de las noticias y su relación con el poder social (PI4 y PI5), esta correlación se produce más con valoraciones utilitarias que emocionales (tablas 11-12). La excepción en este sentido es la Identificación, que se engarza profundamente con el afecto positivo. Asimismo, en la investigación se ha observado que los usuarios comparten noticias que consideran útiles para, de ese modo, lograr casi todos los objetivos de poder social. El único caso en el que esta dimensión de utilidad no es relevante se produce cuando el usuario quiere identificarse con el lector de su noticia. En ese caso cobra importancia el afecto positivo, es decir, el usuario comparte noticias que le han gustado porque cree que su contacto sentirá lo mismo que él.

Desde otra perspectiva, cuando el objetivo consiste en estrechar lazos (bonding social capital), como ocurre con Identificación, el afecto hacia la noticia es más relevante que su potencial utilidad. Si la compartición de noticias se entiende como una especie de recompensa social (Emerson, 1987), como ocurre con Recompensa, o el objetivo es crear nuevos contactos (bridging social capital), entonces el afecto positivo es más relevante como moneda social (Berger, 2014) que la utilidad.

Así, finalmente, se ha encontrado un vínculo entre la intención de educar a otros, la percepción de utilidad de la noticia, las RAA y la preferencia por noticias duras. Cuando los usuarios quieren ilustrar o adoctrinar a sus contactos, eligen compartir en RAA noticias duras que les resultan útiles.

En cuanto a las limitaciones del estudio, este analiza experiencias de usuarios residentes en único país (España). Tomando como punto de partida los resultados presentados, futuros trabajos podrían abordar el mismo objeto en otros países europeos o de América Latina para establecer comparaciones entre ellos. Asimismo, este trabajo se basa en una única metodología, el método de muestreo de experiencias, al ser la primera vez que se analiza este tema en el ámbito español. Por ello, se propone que otras investigaciones utilicen otro tipo de técnicas, como los grupos de discusión con usuarios de redes sociales o las entrevistas en profundidad, con el fin de profundizar y matizar en los motivos y las relaciones identificadas en nuestro análisis cuantitativo.

En este estudio se han considerado variables de control relacionadas con aspectos sociodemográficos como la edad, el nivel económico y el educativo. Sin embargo, no se ha prestado atención a cómo influye el género del usuario que comparte noticias en redes, un tema que también podría ser abordado por otras investigaciones.

Futuros estudios podrían también investigar el uso de las RCS para difundir opiniones consideradas indeseables o impopulares. Si la intención de defender y prescribir opiniones es más común en RAA que en RCS, ello puede implicar que 
el sesgo de confirmación en la compartición de noticias (Shin \& Thorson, 2017) es más fuerte en RAA. La orientación hacia la Legitimación de las RAA amortiguaría incluso el papel de la espiral del silencio, como ya se ha señalado que ocurre en Facebook (Chaudhry \& Gruzd, 2019). Por otro lado, se ha señalado que los filtros burbuja no son tan relevantes como se pensaba en los entornos de las RAA (Dubois \& Blank, 2018). No obstante, este fenómeno podría ser más intenso en las RCS. Es decir, si los usuarios comparten menos contenido con carga ideológica en RCS y autocensuran aquello que interpretan como controvertido, las posibilidades de que encuentren noticias opuestas a sus visiones existentes pueden ser inferiores. En otras palabras, el papel del filtro burbuja y las cámaras de eco, que empiezan a ser cuestionados en el entorno de las RAA, quizá sean más frecuente en las RCS. En este sentido, resulta necesario investigar en el rol de la percepción de heterogeneidad y afinidad de los contactos por parte de los usuarios y su relación con la compartición de noticias.

\section{FINANCIAMIENTO}

Proyecto Consumo de noticias en medios sociales. Análisis de factores en la selección y difusión de contenidos mediáticos (CSO2017-86312-R) financiado por el Ministerio de Economía, Industria y Competitividad (Mineco) de España, la Agencia Estatal de Investigación (AEI) y el Fondo Europeo de Desarrollo Regional (Feder), dentro de la convocatoria 2017 de ayudas a proyectos de I+D+I correspondientes al Programa Estatal de Investigación, Desarrollo e Innovación Orientada a los Retos de la Sociedad en el marco del Plan Estatal de Investigación Científica y Técnica y de Innovación 2013-2016. http://newssharing.es

\section{REFERENCIAS}

An, J., Quercia, D., Cha, M., Gummadi, K., \& Crowcroft, J. (2014). Sharing political news: the balancing act of intimacy and socialization in selective exposure. EPJ Data Science, 3(1), 12. https://doi.org/10.1140/epjds/s13688-014-0012-2

Berger, J. (2014). Word of mouth and interpersonal communication: A review and directions for future research. Journal of Consumer Psychology, 24(4), 586-607.

https://doi.org/10.1016/j.jcps.2014.05.002

Boczkowski, P. J., Matassi, M., \& Mitchelstein, E. (2018). How Young Users Deal With Multiple Platforms: The Role of Meaning-Making in Social Media Repertoires. Journal of ComputerMediated Communication, 23(5), 245-259. https://doi.org/10.1093/jcmc/zmy012 
Chaudhry, I. \& Gruzd, A. (2019). Expressing and Challenging Racist Discourse on Facebook: How Social Media Weaken the "Spiral of Silence" Theory. Policy \& Internet, 12(1), 88-108. https://doi.org/10.1002/poi3.197

Dubois, E. \& Blank, G. (2018). The echo chamber is overstated: the moderating effect of political interest and diverse media. Information, Communication \& Society, 21(5), 729-745. https://doi.org/10.1080/1369118X.2018.1428656

Duffy, A. \& Ling, R. (2019). The Gift of News: Phatic News Sharing on Social Media for Social Cohesion.Journalism Studies, 21(1)1, 72-87. https://doi.org/10.1080/1461670X.2019.1627900

Emerson, R. M. (1987). Toward a theory of value in social exchange. In K. Cook (Ed.), Social Exchange Theory. SAGE.

French Jr., J. R. P. \& Raven, B. (1959). The bases of social power. In D. Cartwright (Ed.), Studies in social power (pp. 150-167). University of Michigan.

Gil-López, T., Shen, C., Benefield, G. A., Palomares, N. A., Kosinski, M., \& Stillwell, D. (2018). One Size Fits All: Context Collapse, Self-Presentation Strategies and Language Styles on Facebook. Journal of Computer-Mediated Communication, 23(3), 127-145. https://doi.org/10.1093/jcmc/zmy006

Gil De Zúñiga, H., Diehl, T., Huber, B., \& Liu, J. (2017). Personality Traits and Social Media Use in 20 Countries: How Personality Relates to Frequency of Social Media Use, Social Media News Use, and Social Media Use for Social Interaction. Cyberpsychology, Behavior, and Social Networking, 20(9), 540-542. https://doi.org/10.1089/cyber.2017.0295

Goncalves, J., Kostakos, V., \& Venkatanathan, J. (2013). Narrowcasting in social media: Effects and perceptions. In Proceedings of the 2013 IEEE/ACM International Conference on Advances in Social Networks Analysis and Mining, ASONAM 2013 (pp. 502-509). ACM. https://doi.org/10.1145/2492517.2492570

Guess, A., Nagler, J., \& Tucker, J. (2019). Less than you think: Prevalence and predictors of fake news dissemination on Facebook. Science Advances, 5(1), eaau4586. https://doi.org/10.1126/sciadv.aau4586

Hasell, A. \& Weeks, B. E. (2016). Partisan Provocation: The Role of Partisan News Use and Emotional Responses in Political Information Sharing in Social Media. Human Communication Research, 42(4), 641-661. https://doi.org/10.1111/hcre.12092

Holton, A. E., Coddington, M., Lewis, S. C., \& Zúñiga, H. G. de. (2015). Reciprocity and the News: The Role of Personal and Social Media Reciprocity in News Creation and Consumption. International Journal of Communication, 9, 2526-2547. Retrieved from http://ijoc.org/index.php/ijoc/article/view/3598

Hossain, M. A., Dwivedi, Y. K., Chan, C., Standing, C., \& Olanrewaju, A. S. (2018). Sharing political content in online social media: A planned and unplanned behaviour approach. Information Systems Frontiers, 20 (3), 485-501. https://doi.org/10.1007/s10796-017-9820-9

Houston, J. B., McKinney, M. S., Thorson, E., Hawthorne, J., Wolfgang, J. D., \& Swasy, A. (2018). The twitterization of journalism: User perceptions of news tweets. Journalism, 21(5), 614-632. https://doi.org/10.1177/1464884918764454

Johnson, B. K. \& Ranzini, G. (2018). Click here to look clever: Self-presentation via selective sharing of music and film on social media. Computers in Human Behavior, 82, 148-158.

https://doi.org/10.1016/j.chb.2018.01.008 
Kalogeropoulos, A., Negredo, S., Picone, I., \& Nielsen, R. K. (2017). Who Shares and Comments on News?: A Cross-National Comparative Analysis of Online and Social Media Participation. Social Media + Society, 3(4). https://doi.org/10.1177/2056305117735754

Kalsnes, B. \& Larsson, A. O. (2019). Facebook News Use During the 2017 Norwegian ElectionsAssessing the Influence of Hyperpartisan News. Journalism Practice, 15(2), 209-225. https://doi.org/10.1080/17512786.2019.1704426

Karapanos, E., Teixeira, P., \& Gouveia, R. (2016). Need fulfillment and experiences on social media: A case on Facebook and WhatsApp. Computers in Human Behavior, 55, 888-897. https://doi.org/10.1016/J.CHB.2015.10.015

Katz, E. (1959). Mass communications research and the study of popular culture: An editorial note on a possible future for this journal. Studies in Public Communication, 2, 1-6. Retrieved from http://repository.upenn.edu/asc_papers/165

Kilgo, D. K., Harlow, S., García-Perdomo, V., \& Salaverría, R. (2018). A new sensation? An international exploration of sensationalism and social media recommendations in online news publications. Journalism, 19(11), 1497-1516. https://doi.org/10.1177/1464884916683549

Kim, E. \& Ihm, J. (2019). More Than Virality: Online Sharing of Controversial News With Activated Audience. Journalism \& Mass Communication Quarterly, 97(1), 118-140. https://doi.org/10.1177/1077699019836950

Knobloch-Westerwick, S. \& Kleinman, S. B. (2012). Preelection Selective Exposure. Communication Research, 39(2), 170-193. hhttps://doi.org/10.1177/0093650211400597

Kubey, R., Larson, R., \& Csikszentmihalyi, M. (1996). Experience Sampling Method Applications to Communication Research Questions. Journal of Communication, 46(2), 99-120. https://doi.org/10.1111/j.1460-2466.1996.tb01476.x

Kümpel, A. S. (2019). The Issue Takes It All? Digital Journalism, 7(2), 165-186. https://doi.org/10.1080/21670811.2018.1465831

Larsson, A. O. (2019). Winning and losing on Social Media-Comparing viral political posts across platforms. Convergence: The International Journal of Research into New Media Technologies, 7(2), 165-186. https://doi.org/10.1002/jnr.490380513

Lee, C. S. \& Ma, L. (2012). News sharing in social media: The effect of gratifications and prior experience. Computers in Human Behavior, 28(2), 331-339. https://doi.org/10.1016/j. chb.2011.10.002

Liu, D. \& Campbell, W. K. (2017). The Big Five personality traits, Big Two metatraits and social media: A meta-analysis. Journal of Research in Personality, 70, 229-240. https://doi.org/10.1016/j.jrp.2017.08.004

Lottridge, D. \& Bentley, F. R. (2018). Let's Hate Together. How People Share News in Messaging, Social, and Public Networks. In Proceedings of the $2018 \mathrm{CHI}$ Conference on Human Factors in Computing Systems - CHI'18 (paper n. 60). ACM Press. https://doi. org/10.1145/3173574.3173634

Lukes, S. (2005). Power: A Radical View. McMillan.

Marwick, A. E. \& boyd, d. (2011). I tweet honestly, I tweet passionately: Twitter users, context collapse, and the imagined audience. New Media \& Society, 13(1), 114-133. https://doi.org/10.1177/1461444810365313 
Noguera-Vivo, J. M. (2018). You get what you give: Sharing as a new radical challenge for journalism. Communication \& Society, 31(4), 147-158. ttps://doi.org/10.15581/003.31.4.147-158

Oeldorf-Hirsch, A. \& Sundar, S. S. (2015). Posting, commenting, and tagging: Effects of sharing news stories on Facebook. Computers in Human Behavior, 44, 240-249. https://doi.org/10.1016/j.chb.2014.11.024

Orellana-Rodriguez, C. \& Keane, M. T. (2018). Attention to news and its dissemination on Twitter: A survey. Computer Science Review, 29, 74-94. https://doi.org/10.1016/J.COSREV.2018.07.001

Ross, C., Orr, E. S., Sisic, M., Arseneault, J. M., Simmering, M. G., \& Orr, R. R. (2009). Personality and motivations associated with Facebook use. Computers in Human Behavior, 25(2), 578-586. https://doi.org/10.1016/J.CHB.2008.12.024

Segado-Boj, F., Díaz-Campo, J., Fernández-Gómez, E., \& Chaparro-Domínguez, M.-Á. (2019). Spanish academics and social networking sites: Use, non-use, and the perceived advantages and drawbacks of Facebook, Twitter, LinkedIn, ResearchGate, and Academia.edu. First Monday, 24(5). https://doi.org/10.5210/fm.v24i5.7296

Segado-Boj, F., Díaz-Campo, J., \& Quevedo-Redondo, R. (2019). Influence of the 'News finds me' Perception on News Sharing and News Consumption on Social Media. Communication Today, 10 (2), 90-104. Retrieved from https://www.communicationtoday.sk/wp-content/ uploads/07.-SEGADO-BOJ-et-al.-\%E2\%80\%93-CT-2-2019.pdf

Shin, J. \& Thorson, K. (2017). Partisan Selective Sharing: The Biased Diffusion of Fact-Checking Messages on Social Media. Journal of Communication, 67(2), 233-255. https://doi.org/10.1111/jcom.12284

Swart, J., Peters, C., \& Broersma, M. (2018). Shedding light on the dark social: The connective role of news and journalism in social media communities. New Media \& Society, 20(11), 4329-4345. https://doi.org/10.1177/1461444818772063

Thompson, N., Wang, X., \& Daya, P. (2019). Determinants of News Sharing Behavior on Social Media. Journal of Computer Information Systems, 60(6), 593-601. https://doi.org/10.1080/08874417.2019.1566803

Waterloo, S. F., Baumgartner, S. E., Peter, J., \& Valkenburg, P. M. (2018). Norms of online expressions of emotion: Comparing Facebook, Twitter, Instagram, and WhatsApp. New Media and Society, 20(5), 1813-1831. https://doi.org/10.1177/1461444817707349

Winter, S., Metzger, M. J., \& Flanagin, A. J. (2016). Selective Use of News Cues: A MultipleMotive Perspective on Information Selection in Social Media Environments. Journal of Communication, 66(4), 669-693. https://doi.org/10.1111/jcom.12241

Witschge, T., Anderson, C., Domingo, D., \& Hermida, A. (2018). Dealing with the mess (we made): Unraveling hybridity, normativity, and complexity in journalism studies. Journalism, 20(5), 651-659. https://doi.org/10.1177/1464884918760669

Yang, C. (2016). Instagram Use, Loneliness, and Social Comparison Orientation: Interact and Browse on Social Media, But Don't Compare. Cyberpsychology, Behavior, and Social Networking, 19(12), 703-708. https://doi.org/10.1089/cyber.2016.0201 


\section{SOBRE LOS AUTORES}

FRANCISCO SEGADO-BOJ, es Profesor Ayudante Doctor en la Universidad Complutense de Madrid. Está acreditado como Profesor Titular de Universidad (desde 2014) y cuenta con dos sexenios de investigación (2004-2009, 2010-2015). Codirige el grupo de investigación "Historia y Estructura de la Comunicación y el Entretenimiento". Su línea de trabajo abarca el periodismo digital, las redes sociales y la comunicación académica. Ha publicado más de treinta artículos en revistas JCR o Scopus como Telematics \& Informatics, Journal of Scholarly Publishing o Comunicar.

* El autor trabaja con dedicación exclusiva en la Universidad Complutense de Madrid desde noviembre de 2019.

iD https://orcid.org/0000-0001-7750-3755

maría-Ángeles ChAPARRo-domínguez, es Profesora Ayudante Doctora en la Universidad Complutense de Madrid. Cuenta con la acreditación de Profesora Titular de Universidad de ANECA y con un sexenio de investigación (2009-2014) reconocido por la CNEAI. Ha sido investigadora invitada en el centro SINTEF de Oslo (Noruega) y ha publicado una treintena de artículos en revistas como Journalism Practice, Global Media and Communication, Young o Nordicom Review. Ha participado en cuatro proyectos de I+D+i con financiación pública. 